\title{
Perforation of tricuspid pouch after tricuspid ring annuloplasty
}

\author{
Mamoru Hamuro, MD, ${ }^{\mathrm{a}}$ Shuji Setozaki, MD, PhD, ${ }^{\mathrm{a}}$ Sakae Enomoto, MD, PhD, ${ }^{\mathrm{a}}$ and Akio Ikai, MD, PhD, ${ }^{\mathrm{b}}$ \\ Shizuoka, Japan
}

\footnotetext{
From the a Department of Cardiovascular Surgery, Okamura Memorial Hospital, Shizuoka, Japan; and ${ }^{\mathrm{b}}$ Department of Cardiovascular Surgery, Mt Fuji Shizuoka Children's Hospital, Shizuoka, Japan.

Disclosures: Authors have nothing to disclose with regard to commercial support.

Received for publication July 16, 2018; revisions received Aug 22, 2018; accepted for publication Sept 3, 2018 ; available ahead of print Oct 22, 2018.

Address for reprints: Mamoru Hamuro, MD, Department of Cardiovascular Surgery, Okamura Memorial Hospital, 293-1 Kakita, Shimizu, Sunto District, Shizuoka Prefecture 411-0904, Japan (E-mail: hmr0705m@ gmail com).

J Thorac Cardiovasc Surg 2019;157:e11-3

$0022-5223 / \$ 36.00$

Copyright (C) 2018 by The American Association for Thoracic Surgery

https://doi.org/10.1016/j.jtcvs.2018.09.012
}

Video clip is available online.

Perforation of a tricuspid pouch after cardiac surgery is rare. There is no clear consensus about surgical intervention for the tricuspid pouch without shunts. We report a case of perforation of the tricuspid pouch causing a left ventricular-right atrial shunt and hemolytic anemia 2 days after tricuspid prosthetic ring annuloplasty. In the case of tricuspid annuloplasty for a patient with a bulging tricuspid pouch without shunts, concomitant ventricular septal defect closure or avoiding the use of prosthetic ring should be considered to prevent the pouch from rupturing.

\section{CLINICAL SUMMARY}

A 75-year-old woman presented with congestive heart failure. Echocardiography revealed severe mitral and moderate tricuspid regurgitation and a subaortic aneurysm projecting from the left ventricle to the right ventricle and right atrium without shunts. Enhanced computed tomography also demonstrated the aneurysm (Figure 1), and the initial diagnosis was aneurysm of the membranous septum (AMS).

Mitral valve repair was performed with a CarpentierEdwards Physio II ring (Edwards Lifesciences Corporation, Irvine, Calif), and tricuspid annuloplasty was performed with an Edwards MC3 tricuspid annuloplasty ring (Edwards Lifesciences Corporation). Intraoperatively, a 20-mm diameter aneurysm was found at the anterior attachment site of the tricuspid septal leaflet in the right atrium. The aneurysm wall was thin, but because there was no shunt, and the aneurysm was located in the thread-free part of the tricuspid

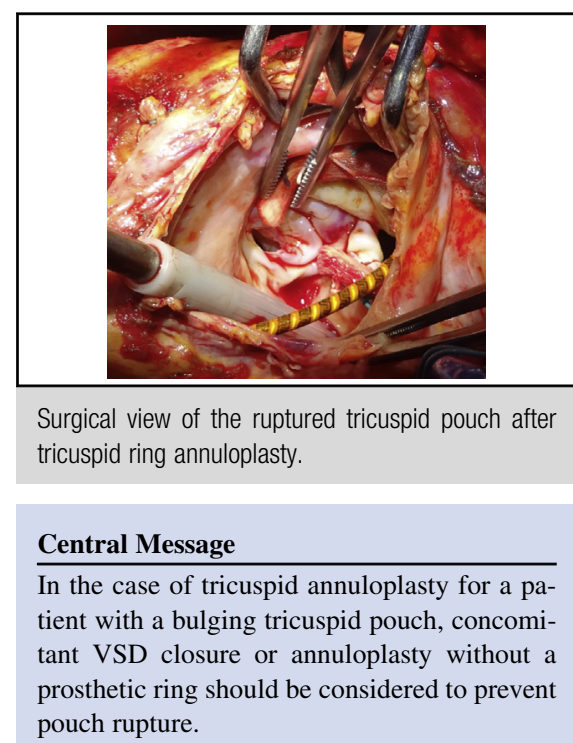

See Editorial Commentary page e15.

annuloplasty, it was left untreated. We performed tricuspid ring annuloplasty while estimating the annulus position on the basis of the attachment site of the septal leaflet. Intraoperative transesophageal echocardiography showed no new shunt around the aneurysm.

The patient was extubated on the operation day, and the immediate postoperative course was uneventful. After postoperative day 2 , however, indirect bilirubin and lactate dehydrogenase levels gradually became elevated, and anemia progressed. Echocardiography and left ventriculography revealed a left ventricular-right atrial shunt around the aneurysm. The diagnosis was confirmed as ruptured AMS as a result of mechanical stimulation by the tricuspid rigid ring and hemolytic anemia.

The reoperation was performed on postoperative day 21 , supported by a congenital heart surgeon. After the cardiopulmonary bypass was set up and the aorta was clamped, the right atrium was opened, revealing a perforation at the aneurysm (Figure 2). Careful observation demonstrated that the tricuspid fibrous annulus was located outside the aneurysm, and the aneurysm included the tricuspid septal leaflet and not the membranous septum. On the basis of these findings, a tricuspid pouch aneurysm was diagnosed instead of AMS. The prosthetic ring was removed, and 


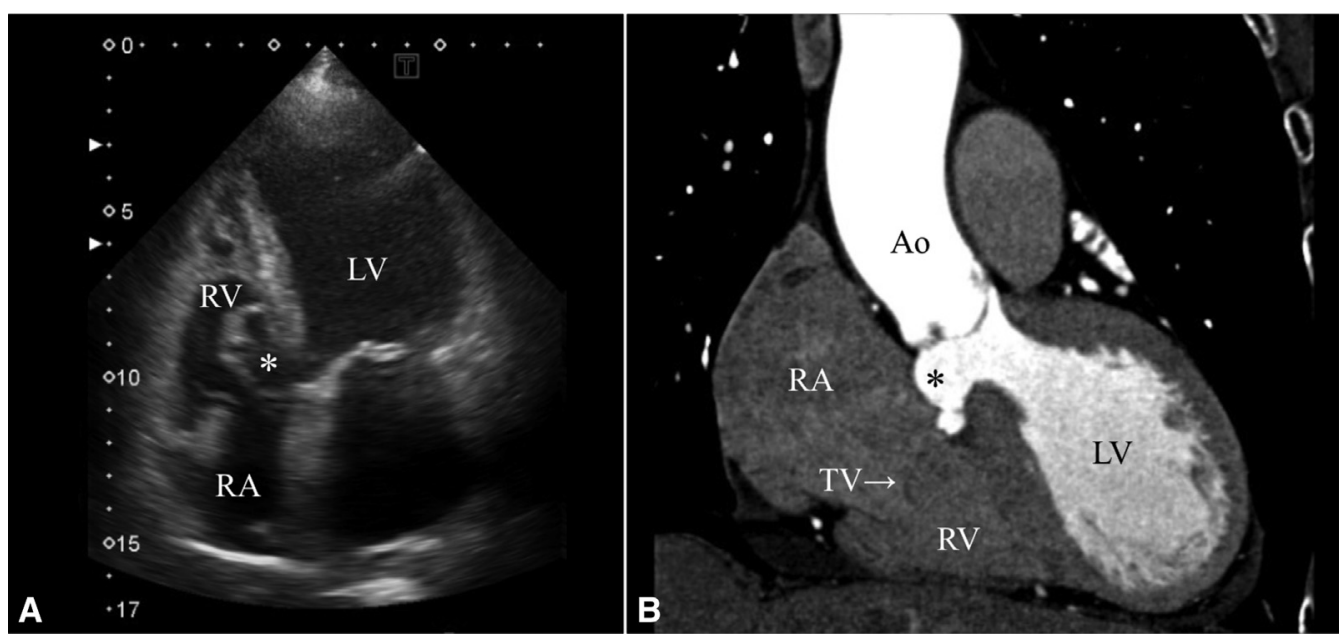

FIGURE 1. Preoperative transthoracic echocardiography (A) and enhanced computed tomography (B) showing subaortic aneurysm (asterisk) projecting from the left ventricle $(L V)$ to the right ventricle $(R V)$ and the right atrium $(R A)$. Ao, Aorta; $T V$, tricuspid valve.

the septal leaflet was partially detached with an incision along the annulus. A $20 \times 15$-mm ventricular septal defect (VSD) with a membranous flap was found under the septal leaflet and closed with a polytetrafluoroethylene patch. Finally, tricuspid ring annuloplasty was repeated. At this time, the attachment portion of the septal leaflet, which was assumed to be the tricuspid annulus during the initial surgery, was confirmed to be more apically located from the fibrous annulus (Video 1). Convalescence was uneventful, and postoperative echocardiography showed trivial tricuspid and mitral regurgitation and no residual shunt.

\section{DISCUSSION}

A tricuspid pouch is a saclike structure projecting toward the right ventricle near the interventricular membranous septum and is involved in spontaneous VSD closure. ${ }^{1}$ It is formed by the adherence of the septal leaflet to the VSD margins. The jet stream through the VSD collides with the tricuspid septal leaflet and causes a pouch structure to protrude into the right ventricle.

The tricuspid pouch can be confused with AMS., ${ }^{2,3}$ Various pathologic theories exist about the mechanism of AMS occurrence, such as membrane arising from the margins of the VSD projected by left ventricular pressure or loss of elastic fiber on the membranous septum. In our case, the aneurysm was initially misdiagnosed as AMS on echocardiography and computed tomography. In particular, the images of preoperative enhanced computed tomography were very similar to those observed in some previous reports of AMS. In addition, during the initial operation, because we estimated the annulus position on

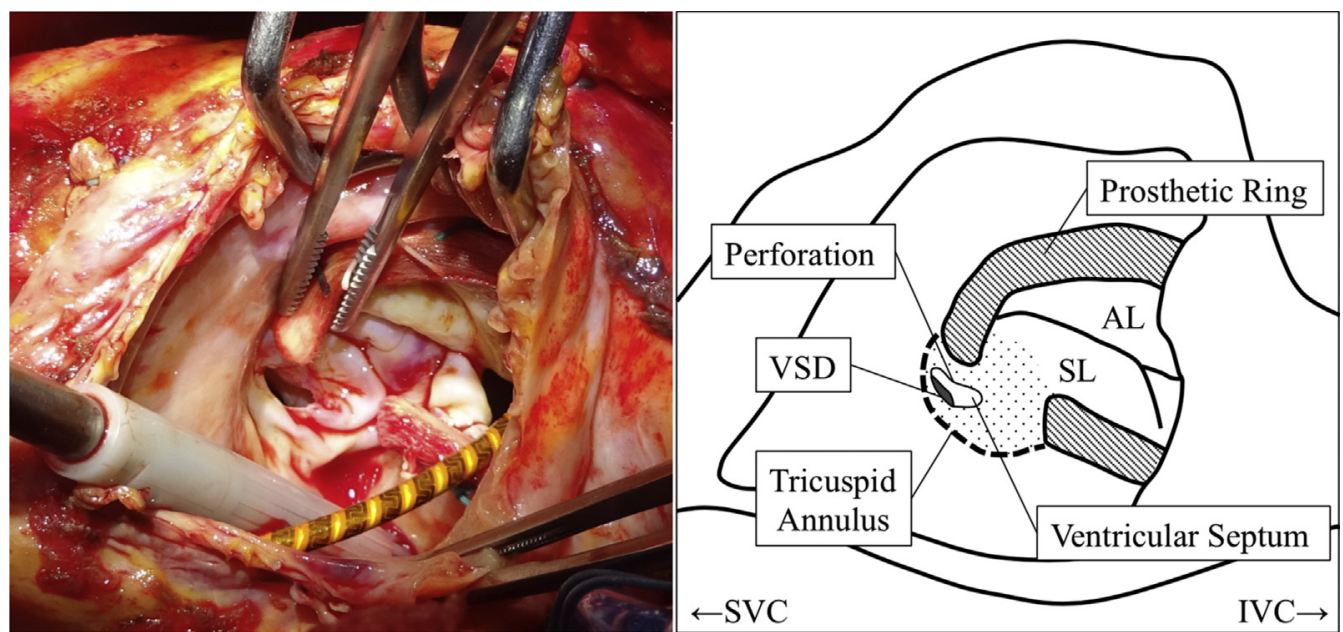

FIGURE 2. Surgical view of the ruptured tricuspid pouch through the right atriotomy during the second operation. The aneurysmlike tricuspid pouch (dot) is located inside the tricuspid annulus. $A L$, Anterior leaflet; $V S D$, ventricular septal defect; $S L$, septal leaflet; $S V C$, superior vena cava; $I V C$, inferior vena cava. 


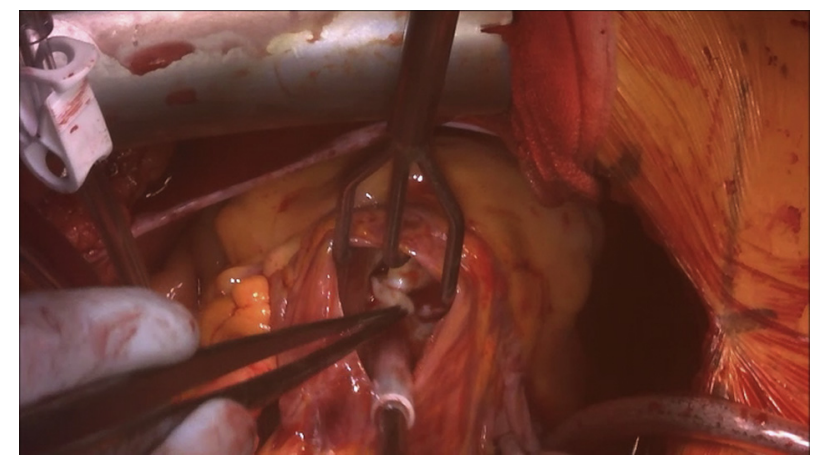

VIDEO 1. Case summary: preoperative and postoperative imaging studies and surgical findings. $C T$, Computed tomography; $L V$, left ventricle; $R V$, right ventricle; $R A$, right atrium; $L V G$, left ventriculography; $V S D$, ventricular septal defect; $T R$, tricuspid regurgitation. Video available at: https:// www.jtcvs.org/article/S0022-5223(18)32511-X/fulltext.

the basis of the leaflet hinge, we did not identify true annular position. During the second operation, however, a tricuspid annulus located outside the aneurysm and a membranous flap in the rim of the VSD were identified. The final diagnosis was therefore a tricuspid pouch. The aneurysmal structure projecting to the right atrium was a part of the septal leaflet, which had been caused to bulge by the left ventricular pressure through the large VSD. Between the septal leaflet hinge and tricuspid fibrous annulus, a gap wider than usual was observed. Chesler and colleagues ${ }^{4}$ reported abnormal, short, and tethered chordae of tricuspid septal leaflets in patients with VSD and tricuspid pouch. In cases of tricuspid pouch, the septal leaflet may partially attach more apically than usual as a result of the process of natural closure of the VSD. Moreover, with tricuspid pouch projecting to the right atrium, the fibrous tissue of the tricuspid annulus may not be easy to identify. The position of the annulus may be misinterpreted even with intraoperative observation; it is therefore important to ascertain the annular position on the basis of careful observation not only of the leaflet hinge but also of the fibrous annulus.

Tricuspid annuloplasty for patients with a bulging tricuspid pouch in the absence of residual shunts requires careful consideration. The clinical course of most cases of tricuspid pouch without shunts is uneventful, and complications such as rupture, endocarditis, and thrombosis are unusual. ${ }^{5}$ Tricuspid pouch perforation after cardiac surgery has rarely been reported. No clear consensus exists about surgical intervention of tricuspid pouches without shunts even with concomitant surgical procedures. In our case, the postoperative tricuspid pouch rupture may have been due to mechanical stress caused by the tricuspid rigid ring. It might have been better to close VSD at the initial operation or to perform tricuspid annuloplasty with a soft material such as autologous pericardium or suture annuloplasty to avoid interference with the bulging tricuspid pouch.

\section{CONCLUSIONS}

During tricuspid annuloplasty in a patient with a bulging tricuspid pouch without shunts, it is important to consider concomitant VSD closure or annuloplasty without using a prosthetic ring to prevent tricuspid pouch perforation.

\section{References}

1. Zhang J, Ko JM, Guileyardo JM, Roberts WC. A review of spontaneous closure of ventricular septal defect. Proc (Bayl Univ Med Cent). 2015;28: 516-20.

2. Idriss FS1, Muster AJ, Paul MH, Backer CL, Mavroudis C. Ventricular septal defect with tricuspid pouch with and without transposition. Anatomic and surgical considerations. J Thorac Cardiovasc Surg. 1992;103:52-9.

3. Abdul Jabbar A, Mufti O, Mazur W, Quraishi MB, Srivastava BK, Tivakaran V. Isolated aneurysms of the membranous ventricular septum without residual shunts: systematic review and description of 3 cases in adults. J Ultrasound Med. 2017;36:869-78

4. Chesler E, Korns ME, Edwards JE. Anomalies of the tricuspid valve, including pouches, resembling aneurysms of the membranous ventricular septum. Am J Cardiol. 1968;21:661-8.

5. Yamamoto K, Ito H, Hiraiwa T. Perforation of a tricuspid pouch caused by infective endocarditis. Ann Thorac Surg. 2008;86:1670-2. 